02,13

\title{
Сверхпроводящие микроструктуры с высоким импедансом
}

\author{
(C) К.В. Шеин ${ }^{1}$, А.А. Заруднева ${ }^{1}$, В.О. Емельянова ${ }^{1}$, М.А. Логунова ${ }^{1}$, В.И. Чичков ${ }^{2}$, А.С. Соболев ${ }^{3}$, \\ B.В. Завьялов ${ }^{1,4}$, J.S. Lehtinen ${ }^{5,6}$, Е.О. Смирнов ${ }^{7}$, Ю.П. Корнеева ${ }^{7}$, А.А. Корнеев ${ }^{7,1}$, К.Ю. Арутюнов ${ }^{1,4, \uparrow}$ \\ ${ }^{1}$ Национальный исследовательский университет „Высшая школа экономики“, \\ Москва, Россия \\ ${ }^{2}$ Национальный исследовательский технологический университет „МИСиС“, \\ Москва, Россия \\ ${ }^{3}$ Институт радиотехники и электроники (ИРЭ) им. В.А. Котельникова РАН, \\ Москва, Россия \\ ${ }^{4}$ Институт фоизических проблем им. П.Л. Капицы РАН, \\ Москва, Россия \\ ${ }^{5}$ VTT Technical Research Centre of Finland Ltd., \\ Espoo, 02150 Finland \\ ${ }^{6}$ Department of Physics, University of Jyvaskyla, \\ PB 35, Jyvaskyla, Fl-40014 Finland \\ ${ }^{7}$ Московский педагогический государственный университет, \\ Москва, Россия \\ ฯ E-mail: karutyunov@hse.ru
}

Поступила в Редакцию 26 марта 2020 г.

В окончательной редакции 26 марта 2020 г.

Принята к публикации 2 апреля 2020 г.

При сверхнизких температурах были исследованы транспортные свойства двух типов квазиодномерных сверхпроводящих микроструктур: тонкие каналы, плотно упакованные в форме меандра, и цепочки туннельных контактов сверхпроводник-изолятор-сверхпроводник. Оба типа микроструктур продемонстрировали высокое значение высокочастотного импеданса и/или динамического сопротивления. Исследование открывает возможность использовать такие структуры в качестве стабилизирующих ток балластных элементов с нулевой величиной диссипации.

Ключевые слова: сверхпроводимость, тонкие пленки, кинетическая индуктивность, туннельные контакты, высокий импеданс.

DOI: 10.21883/FTT.2020.09.49756.01H

\section{1. Введение}

Последние годы наблюдается замедление роста степени интеграции коммерческих микро- и наноэлектронных устройств, формально знаменующее нарушение закона Мура [1]. Тому можно привести две причины: большое тепловыделение на единицу объема (или площади) и различные квантовые размерные эффекты. Радикальным решением первой проблемы может быть переход в критических элементах схемы от нормальных металлов или полупроводников к сверхпроводящим материалам. Наряду с этим тривиальным подходом, использование сверхпроводящей логики RSFQ (rapid single flux quantum) позволяет не только значительно снизить энергозатраты, но и добиться быстродействия, на два порядка превосходящего стандартные решения на базе CMOS (complementary metal-oxide semiconductor) технологии [2]. Ожидается, что элементная база квантовых компьютеров также может быть построена с использованием сверхпроводящих материалов.

Одним из факторов, ограничивающих быстродействие сверхпроводящих наноэлектронных устройств, является их высокая кинетическая индуктивность. Однако в ряде приложений эффект кинетической индуктуивности может быть полезен. В последние годы возрос интерес к физике квазиодномерных сверхпроводников [3], в которых флуктуации параметра порядка $\Delta=|\Delta| e^{i \phi}$ могут играть существенную роль. В частности, было показано, что специфическое проявление квантовых флуктуаций - квантовое проскальзывание фазы - дуально джозефсоновскому туннелированию [4-7]. Фаза параметра порядка $\phi$ и квазизаряд $q$ являются комплексносопряженными величинами. Следовательно, для высокой неопределенности фазы (=сильных флуктуаций) необходимо зафиксировать заряд, что для электронной схемы эквивалентно стабилизации тока, являющегося производной заряда по времени $I \equiv d q / d t$. Стандартное решение с использованием балластных резисторов [8-10] не лишено недостатков. В частности, наличие высокоомных элементов в цепи неизбежно приводит к присутствию джонсоновского шума [11].

В настоящей статье мы исследуем работу двух типов микроэлектронных структур: тонких сверхпроводящих каналов с существенной величиной кинетической индуктивности и цепочек туннельных контактов сверхпроводник-изолятор-сверхпроводник (СИС). Обе 
системы на переменном токе демонстрируют высокий импеданс (при нулевой диссипации), и могут быть использованы как стабилизирующие ток элементы электронных цепей.

\section{2. Теория}

В рамках стандартных моделей сверхпроводимости могут быть получены частотные и температурные зависимости кинетической индуктивности $L_{k}[12]$. С использованием уравнений Гинзбурга-Ландау, для температур, близких к критической $T_{c}$, и при малом токе смещения $I \rightarrow 0$ можно получить следующую температурную зависимость

$$
L_{k}(T)=\frac{L_{k}(0)}{1-\left(T / T_{c}\right)} .
$$

Микроскопическая модель Бардина-Купера-Шриффера дает простое выражение для кинетической индуктуивности тонкого сверхпроводящего канала

$$
L_{k}=\frac{\hbar R_{N}}{p i \Delta(T)} \frac{1}{\tanh \left\{\frac{\Delta(T)}{2 k_{B} T}\right\}} .
$$

Т.е. для получения существенных значений кинетической индуктивности в области низких температур $T \ll T_{c}$ предпочтительны сверхпроводящие материалы с высоким значением сопротивления в нормальном состоянии $R_{N}$ и малой величиной критической температуры $T_{c}$. При включении в цепь переменного тока не очень высокой частоты $f<\Delta / h$ импеданс такого элемента $Z_{L}(f) \sim f L$ может достигать вполне значительной величины, обладая при этом нулевой диссипацией по постоянной составляющей $[13,14]$.

Альтернативным к высокой кинетической индуктивности решением может быть использование туннельных СИС-элементов, обеспечивающих высокое динамическое сопротивление $R_{\text {dyn }} \equiv d V / d I \gg R_{N}$ в области малых токов, т.е. до полного выхода вольт-амперной характеристики (BAX) на квазичастичную ветвь [15]. Зависимость динамического сопротивление от тока $R_{\mathrm{dyn}}(I)$ является существенно нелинейной и стремится к бесконечности при нулевых смещениях $I \rightarrow 0$.

\section{3. Образцы и методика эксперимента}

Методом фотолитографии и вакуумного напыления были изготовлены микроструктуры, представляющие собой длинные и тонкие каналы, плотно упакованные в форме меандра (рис. 1, вставка).

Материалами для сверхпроводящих структур были выбраны $\mathrm{NbN}$ и Ti. Толщина и ширина $d / w$ пленок составляла $5 / 100 \mathrm{~nm}$ и $30 \mathrm{~nm} / 2 \mu \mathrm{m}$ соответственно. Сопротивление на квадрат в нормальном состоянии составило $R_{N \square}(\mathrm{NbN}) \approx 950 \Omega$ и $R_{N \square}(\mathrm{Ti})=200 \Omega$, соответственно. Сечения нанопроводов были выбраны заведомо больше тех размеров, где вклад термических

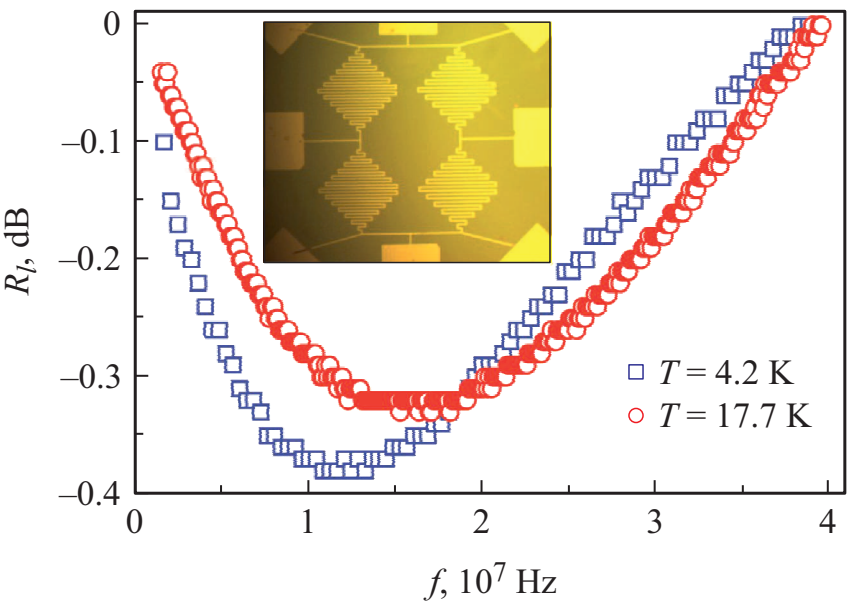

Рис. 1. Зависимости величины высокочастотного коэффициента отражения $R_{l}$ от частоты $f$ для микроструктуры из $\mathrm{NbN}$ с длиной $l=50 \mu \mathrm{m}$, толщиной $d=5 \mathrm{~nm}$ и шириной линии $w=100 \mathrm{~nm}$, упакованной в область $10 \times 10 \mu \mathrm{m}$, при температуре выше и ниже критической $T_{c}(\mathrm{NbN})=6.5 \mathrm{~K}$. На вставке приведена микрофотография типичной структуры из титана: тонкая сверхпроводящая полоска, упакованная в форме меандра.

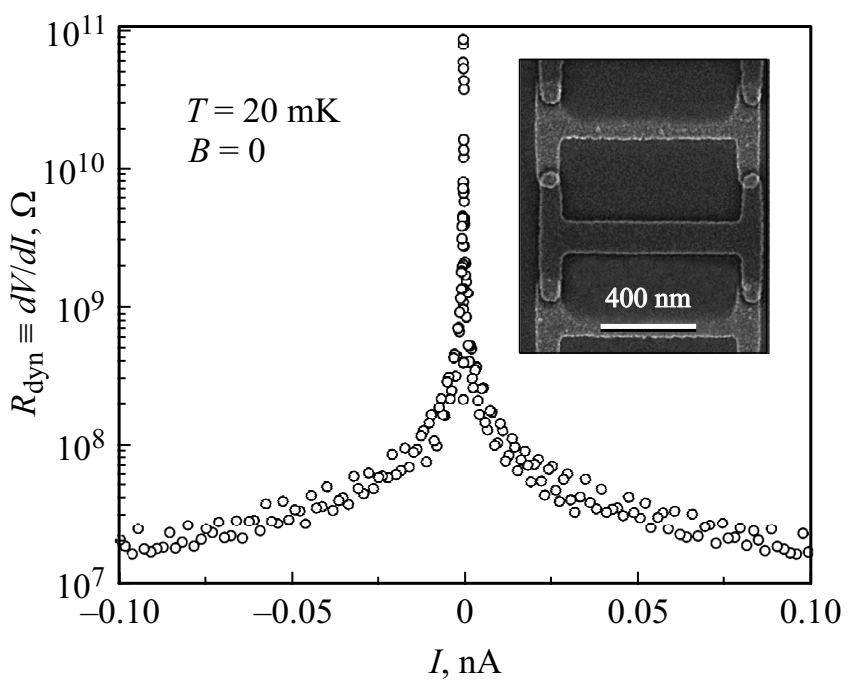

Рис. 2. Зависимость динамического сопротивления $R_{\text {dyn }} \equiv d V / d I$ от тока смещения $I$ для цепочки из 25 пар туннельных контактов $\mathrm{Al} / \mathrm{AlO}_{x} / \mathrm{Al} / / \ldots / \mathrm{AlO}_{x} / \mathrm{Al}$ (вставка) в нулевом магнитном поле и при сверхнизкой температуре $T=20 \mathrm{mK} \ll T_{c}(\mathrm{Al})=1.2 \mathrm{~K}$.

и/или квантовых флуктуаций существенен для Ті [16-20] и $\mathrm{NbN}$ [21]. Зависимости сопротивления от температуры обнаружили четкие сверхпроводящие переходы в области $T_{c}(\mathrm{NbN})=6.5 \pm 0.5 \mathrm{~K}$ и $T_{c}(\mathrm{Ti})=0.42 \pm 0.02 \mathrm{~K}$. Никаких немонотонностей формы $R(T)$ перехода, которые могут свидетельствовать либо о неоднородности системы [22], либо о ее неравновесности [23,24], обнаружено не было. 
Методом электронной взрывной литографии и многоуглового вакуумного напыления были изготовлены наноструктуры, представляющие собой цепочки идентичных элементов $\mathrm{Al} / \mathrm{AlO}_{+} x / \mathrm{Al} / \ldots / \mathrm{AlO}_{x} / \mathrm{Al}$ (рис. 2, вставка) с критической температурой сверхпроводящего алюминия $T_{c}(\mathrm{Al})=1.2 \mathrm{~K}$.

Все эксперименты проводились при низких и сверхнизких температурах. Измерения зависимостей $R(T)$ и $V(I)$ делались с использованием многоступенчатой системы $R L C$-фильтров, снижающих влияние электромагнитных наводок [25]. Измерения ВАХ и амплитудночастотных характеристик (АЧХ) проводилось стандартным четырехконтактным способом.

\section{4. Результаты и обсуждение}

Для измерения ВАХ и АЧХ квазиодномерных сверхпроводящих каналов, ток в цепи и напряжение на образце регистрировалось одновременно. Выход высокочастотного генератора был нагружен через $50-\Omega$ криогенный коаксиальный кабель на исследуемую $L C$-цепь, где $L-$ неизвестная индуктивность микроструктуры, а $C=50 \mathrm{pF}$ - известная величина емкости параллельно подключенного балластного конденсатора. Отдельные измерения конденсатора показали, что его емкость не сильно меняется от комнатной температуры до $2 \mathrm{~K}$ на частотах до $400 \mathrm{MHz}$. В эксперименте измерялся $($ в $\mathrm{dB})$ коэффициент отражения $R_{l}$ как функция частоты зондирующего сигнала

$$
R_{l}=-20 \log _{10}\left|\frac{Z_{\text {load }}-Z_{\text {source }}}{Z_{\text {load }}+Z_{\text {source }}}\right|,
$$

где $Z_{\text {load }}$ и $Z_{\text {source }}$ - импедансы нагрузки и источника соответственно. Зависимости $R_{l}(f)$ наглядно демонстрируют минимум, соответствующий резонансной частоте $f_{\text {res }}$ системы (рис. 1). Сдвиг $f_{\text {res }}$ к более низким значениям при охлаждении системы до сверхпроводящего состояния указывает на увеличение импеданса системы. Учитывая, что балластная емкость постоянна, $C=50 \mathrm{pF}$, значение общей индуктивности можно определить тривиально: $L=L_{k}+L_{m}=\left(1 / 4 \pi^{2} C\right)\left(1 / f_{\text {res }}\right)^{2}$. В нашем случае сверхпроводящей системы микронных размеров магнитная индуктивность $L_{m}$ существенно меньше кинетической и определяется исключительно геометрией структуры, и, следовательно, одинакова в нормальном и сверхпроводящем состояниях. Для тонкопленочного меандра $\mathrm{NbN}$ с длиной $l=500 \mu \mathrm{m}$, толщиной $d=5 \mathrm{~nm}$ и шириной линии $w=100 \mathrm{~nm}$, упакованного в область $10 \times 10 \mu \mathrm{m}$, используя зависимости $R_{l}(f)$ (рис. 1), можно оценить индуктивность $L_{k} \approx 5 \mu \mathrm{H}$ при $T=4.2 \mathrm{~K}$. Соответствующая кинетическая индуктивность на единицу площади в сверхпроводящем состоянии $L_{k \square} \approx 0.75 \mathrm{nH} / \square$. Наши результаты по структурам из $\mathrm{NbN}$ находятся в разумном количественном согласии с литературными данными [12-14]. Несколько более высокие значения удельной кинетической индуктивности по сравнению с $[12,26]$ можно объяснить увеличением беспорядка в исследованных пленках $\mathrm{NbN}$, что приводит к более высокому удельному сопротивлению $\left(R_{\square} \approx 1 \mathrm{k} \Omega\right.$ против $875 \Omega)$ и более низкой критической температуре $\left(T_{c}=6.5 \mathrm{~K}\right.$ против $\left.10 \mathrm{~K}\right)$. Варьирование измерительного тока в широких пределах не влияет на зависимость $R_{l}(f)$. На частотах порядка $1 \mathrm{GHz}$ высокочастотный импеданс достигает величины $Z_{L}(f) \approx 30 \mathrm{k} \Omega$, что не является таким уж и „выдающимся“ результатом. Однако современными литографическими методами вполне реалистично изготовление наноструктур с примерно стократно большим значением кинетической индуктивности без существенного шунтирования паразитной емкостью. Соответствующий высокочастотный импеданс мегаомного диапазона уже вполне достаточен для исследования квантовой динамики заряда в широких частотных и токовых диапазонах [9,10,27]. Качественно аналогичные результаты были получены и на титановых микроструктурах (рис. 1, вставка). Но специфика эксперимента в рефрижераторе растворения ${ }^{3} \mathrm{He}^{4} \mathrm{He}$ при сверхнизких температурах $T<100 \mathrm{mK}$ привела к существенно большей ошибке измерений $Z_{L}(f)$ для титана. Вероятно, эффект связан с перегревом тестируемых структур зондирующим сигналом.

При сверхнизких температурах $T \sim 20 \mathrm{mK} \ll T_{c}(\mathrm{Al})$ $=1.2 \mathrm{~K}$ исследовались транспортные свойства цепочек $\mathrm{Al} / \mathrm{AlO}_{x} / \mathrm{Al} / \ldots / \mathrm{AlO}_{x} / \mathrm{Al}$ (рис. 2, вставка). Параметры окисления алюминиевой пленки выбирались таким образом, чтобы толщина туннельного барьера оказалась достаточно большой, и, как следствие, величина джозефсоновского ток - малой: $I_{c}<10$ рА. Соответствующая энергия $E_{J}=\hbar I_{c}$ много меньше зарядовой энергии $E_{c}=e^{2} / 2 C$, где $C$ может быть оценена как емкость последовательно соединенных плоских конденсаторов с толщиной диэлектрик $\sim 2 \mathrm{~nm}$, площадью обкладок (перекрытие контактов) $\sim 100 \times 100 \mathrm{~nm}$ и с диэлектрической проницаемостью окиси алюминия $\varepsilon \sim 10$. Условие $E_{J} \ll E_{c}$ приводит к доминированию зарядовых эффектов над джозефсоновскими [28], и, соответственно, позволяет пренебречь квантовыми флуктуациями.

Bce структуры $\mathrm{Al} / \mathrm{AlO}_{x} / \mathrm{Al} / \ldots \mathrm{AlO}_{x} / \mathrm{Al}$ продемонстрировали ВАХ, типичные для последовательно соединенных туннельных СИС-контактов. Динамическое сопротивление $R_{\mathrm{dyn}} \equiv d V / d I$, полученное численным дифференцированием ВАХ, обнаруживает четкую сингулярность при малых токовых смещениях достигая величины $R_{\text {dyn }}(I \rightarrow 0) \sim 10^{11} \Omega$ (рис. 2 ).

При больших значениях тока динамическое сопротивление падает, стремясь к величине $R_{\text {dyn }}(I \gg 0) \sim 10^{5} \Omega$, соответствуя туннельному сопротивлению квазичастичной ветви ВАХ. Последнее наблюдение ограничивает использование СИС-контактов в качестве стабилизирующих ток элементов. Действительно, исследование квантовой динамики заряда с использованием цепочек СИС-контактов позволило зарегистрировать четкую кулоновскую блокаду, тогда как при конечных токах блоховские сингулярности сильно размываются даже по 
сравнению со случаем чисто диссипативных токовых электродов, изготовленных из высокоомных материалов [27]. По всей видимости, при конечных смещениях преимущество должно быть отдано сверхпроводящим токосмещающим элементам с высокой кинетической индуктивностью.

\section{5. Заключение}

Были исследованы транспортные свойства двух типов квазиодномерных сверхпроводящих микроструктур: тонкие каналы с высокой величиной кинетической индуктивности и цепочки туннельных СИС-контактов. Высокая величина динамического сопротивления СИСконтактов при малых токах делает их исключительно эффективными для наблюдения таких эффектов, как кулоновская блокада на ВАХ квазиодномерных сверхпроводящих каналов в режиме квантовых флуктуаций параметра порядка [18]. Однако сильная нелинейность ВАХ СИС-контактов приводит к существенному падению динамического сопротивления при конечных токах. Напротив, тонкие и длинные сверхпроводящие элементы обладают высокой величиной кинетической индуктивности, которая в области конечных, но не очень больших токов $I \ll I_{c}$ позволяет достигать на достаточно высоких частотах значительной величины импеданса, обеспечивая тем самым эффективную стабилизацию тока.

\section{Финансирование работы}

Постановка задачи, анализ результатов и подготовка текста статьи проводились КЮА и были поддержаны грантом № 19-01-050 в рамках Программы «Научный фонд Национального исследовательского университета „Высшая школа экономики“ (НИУ ВШЭ)» в 2019-2020],гг. и в рамках государственной поддержки ведущих университетов Российской Федерации , ,5-100“.

\section{Конфликт интересов}

Авторы заявляют, что у них нет конфликта интересов.

\section{Список литературы}

[1] G. Moore. Electronics 38, 8, 114 (1965).

[2] K.K. Likharev, V.K. Semenov. IEEE Trans. Appl. Supercond. 1, 3 (1991).

[3] K.Yu. Arutyunov, D. Golubev, A.D. Zaikin. Phys. Rep. 464, 1 (2008).

[4] A.D. Zaikin, S.V. Panyukov. Phys. Lett. A 120, 306 (1987).

[5] D.V. Averin, A.A. Odintsov. Phys. Lett. A 140, 251 (1989).

[6] A.D. Zaikin. J. Low Temp. Phys. 80, 223 (1990).

[7] J.E. Mooij, Y.V. Nazarov. Nat. Phys. 2, 169 (2006).

[8] L.S. Kuzmin, D.S. Havil. Phys. Rev. Lett. 67, 2890 (1991).

[9] T.T. Hongisto, A.B. Zorin. Phys. Rev. Lett. 108, 9, 097001 (2012).
[10] J.S. Lehtinen, K. Zakharov, K.Yu. Arutyunov. Phys. Rev. Lett. 109, 18, 187001 (2012).

[11] C.H. Webster, J.C. Fenton, T.T. Hongisto, S.P. Giblin, A.B. Zorin, P.A. Warburton. Phys. Rev. B 87, 144510 (2013).

[12] A.J. Annunziata. PhD Thesis, Yale University (2010).

[13] A.J. Kerman, E.A. Dauler, W.E. Keicher, J.K.W. Yang, K.K. Berggren, G. Gol'tsman, B.M. Voronov. Appl. Phys. Lett. 88, 11, 111116 (2006).

[14] A.J. Kerman, E.A. Dauler, J.K.W. Yang, K.M. Rosfjord, V. Anant, K.K. Berggren, G. Gol'tsman, B.M. Voronov. Appl. Phys. Lett. 90, 10, 101110 (2007).

[15] M. Tinkham. Introduction to Superconductivity. 2nd ed. McGraw-Hill, N. Y.(1996).

[16] J.S. Lehtinen, T. Sajavaara, K.Yu. Arutyunov, M.Yu. Presnjakov, A. Vasiliev. Phys. Rev. B 85, 094508 (2012).

[17] K.Yu. Arutyunov, J.S. Lehtinen, T.J. Rantala. J. Supercond. Nov. Magn. 29, 569 (2016).

[18] K.Yu. Arutyunov, J.S. Lehtinen. Nanoscale Res. Lett. 11, 364 (2016).

[19] K.Yu. Arutyunov, J.S. Lehtinen. Physica C: 533, 158 (2017).

[20] K.Yu. Arutyunov, J.S. Lehtinen, A.A. Radkevich, A.G. Semenov, A.D.Zaikin. J. Magn. Magn. Mater. 459, 356 (2018).

[21] K.Yu. Arutyunov, A. Ramos-Álvarez, A.V. Semenov, Yu.P. Korneeva, P.P. An, A.A. Korneev, A. Murphy, A. Bezryadin, G.N. Gol'tsman. Nanotechnol. 27, 47LT021 (2016).

[22] K.Yu. Arutyunov, S.V. Lotkhov, A.B. Pavolotski, D.A. Presnov, L. Rinderer. Phys. Rev. B 59, 9, 6487 (1999).

[23] K.Yu. Arutyunov. Phys. Rev. B 53, 18, 12304 (1996).

[24] K.Yu. Arutyunov, T.V. Ryynanen, J.P. Pekola, A.B. Pavolotski. Phys. Rev. B 63, 9, 092506 (2001).

[25] V.V. Zavyalov, S.A. Chernyaev, K.V. Shein, A.G. Shukaleva, K.Yu. Arutyunov. J. Phys.: Conf. Ser. 969, 012086 (2018).

[26] A.J. Annunziata, D.F. Santavicca, L. Frunzio, G. Catelani, M.J. Rooks, A. Frydman, D.E. Prober. Nanotechnol. 21, 445202 (2010).

[27] Z.M. Wang, J.S. Lehtinen, K.Yu. Arutyunov. Appl. Phys. Lett. 114, 242601 (2019).

[28] G. Schön, A.D. Zaikin. Phys. Rep. 198, 237 (1990).

Редактор Е.В. Толстякова 